\title{
Fırçasız Doğru Akım Motorlarının Hız Kontrolünü Gerçekleştirmek İçin PID/PD Kontrolcü Tasarımı ve Performans İncelemesi
}

\author{
Abdülsamed Tabak ${ }^{1 *}$ \\ ${ }^{1}$ Necmettin Erbakan Üniversitesi, Mühendislik ve Mimarlık Fakültesi, Mekatronik Mühendisliği Bölümü, Konya, Türkiye (ORCID: 0000-0001-8832-6408)
}

(İlk Geliş Tarihi 21 Mart 2020 ve Kabul Tarihi 23 Mayıs 2020)

(DOI: 10.31590/ejosat.707004)

ATIF/REFERENCE: Tabak, A. (2020). Fırçasız Doğru Akım Motorlarının Hız Kontrolünü Gerçekleştirmek İçin PID/PD Kontrolcü Tasarımı ve Performans İncelemesi. Avrupa Bilim ve Teknoloji Dergisi, (19), 145-155.

$\ddot{O} \mathbf{z}$

Fırçasız doğru akım (FDA) motorlarının kullanımı, düzgün mekanik tork sağlaması ve yüksek güç yoğunluğuna sahip olması gibi avantajlarından dolayı günden güne artmaktadır. FDA motorlarının kullanımı yaygınlaştıkça bu motorların kontrolü ile ilgili yeni çalışmalar ortaya konmaktadır. Bizim çalışmamızda, çıkıştaki maksimum aşma miktarı ve oturma zamanı gibi değerlerin düşürülmesi amacıyla ilk olarak FDA motorun PID kontrolü yapılmış ve kontrolcünün parametreleri Balina Optimizasyon Algoritması (BOA) ile elde edilmiştir. Elde edilen sonuçlar literatürde aynı parametrelere sahip FDA motorun genetik algoritma (GA), parçacık sürü optimizasyonu (PSO), LQR ve LQ yöntemleri ile optimize edilen PID kontrolcülerinin kullanıldığı sistemin sonuçları ile karşılaştırılmıştır. Bunun yanında PID yerine PID/PD kontrolcü kullanılarak aynı sistemin kontrolü tekrar yapılmış ve parametrelerin belirlenmesi için yine BOA'dan faydalanılmıştır. PID/PD kontrolcü ile tasarlanan sistem; maksimum aşma, oturma zamanı ve yükselme zamanı gibi performans kriterleri açısından PID kontrolcülü sistemlerle karşılaştırılmıştır. Ardından hem PID hem de PID/PD kontrolcülerin dinamik testini gerçekleştirmek amacıyla motorun hızı artırılarak sonuçlar elde edilmiş, irdelenmiş ve birbirleri ile karşılaş̧ırılmıştır. Sonuçlara bakıldığında ise BOA-PID’nin diğer yöntemlerle elde edilen PID sonuçlarından daha iyi performans gösterdiği, BOA-PID/PD’nin ise PID’nin kullanıldığı tüm çalışmalardan daha iyi performans sergilediği görülmüsstür.

Anahtar Kelimeler: Fırçasız DA motoru, PID kontrol, PID/PD kontrol, Balina optimizasyon algoritması, Parametrelerin belirlenmesi.

\section{Design and Performance Analyze of PID/PD Controller to Perform Speed Control of Brushless DC Motors}

\begin{abstract}
The usage of brushless DC motors (BLDC) is increasing day by day due to its advantages such as providing constant mechanical torque and high power density. As the use of BLDC motors becomes widespread, a number of new studies have been introduced in terms of control of these motors In our study, firstly the PID control of the BLDC motor was performed to reduce the values such as the maximum overshoot and the settling time at the output, and the controller's parameters were obtained by the Whale Optimization Algorithm (WOA). The results were compared with the results of the system with the same parameters of the BLDC motor in the literature using PID controllers optimized by the genetic algorithm (GA), particle swarm optimization (PSO), LQR and LQ methods. In addition, PID/PD controller was utilized instead of PID for the same system and WOA was again used to determine parameters. The system designed with PID/PD controller has been compared with the system designed with PID controller systems in terms of performance criteria such as maximum overshoot, settling time and rise time. Then, in order to perform the dynamic test of both PID and PID/PD controllers, the speed of the motor was increased and the results were obtained, examined and compared with each other.
\end{abstract}

* Sorumlu Yazar: Necmettin Erbakan Üniversitesi, Mühendislik Fakültesi, Mekatronik Mühendisliği Bölümü, Konya, Türkiye, ORCID: 0000-00018832-6408, atabak@erbakan.edu.tr 
As a result, it was observed that BOA-PID performed better than PID results obtained by other methods, while BOA-PID PD performed better than all studies using PID.

Keywords: Brushless DC motor, PID control, PID/PD control, Whale optimization algorithm, Determination of parameters.

\section{Giriş}

Fırçasız DA (FDA) motorlar; yüksek verimlilik, sessiz çalışma, uzun ömürlü olma, daha az bakım gerektirme, sabit tork sağlama, geniş hız ve tork kontrol aralığına sahip olma gibi özelliklerinden dolayı son yıllarda endüstri, otomotiv, askeri ekipmanlar, evsel cihazlar ve otomasyon sistemlerinde yaygın halde kullanılmaktadır (Grigorie, Khan, Botez, Mamou, \& Mébarki, 2019; Lu, Zhang, \& $\mathrm{Qu}, 2008)$. Firçalı motorlardaki komütasyon işleminde yardımcı olan firçalar zamanla aşınarak motorun bozulması, verim kaybı ve kıvılcım oluşturması gibi birtakım sorunlara neden olmaktadır. Fırçasız DA motorlarda komütasyon işlemi mekanik olmak yerine Hall sensörleri ve güç elektroniği tabanlı anahtarlama devreleri sayesinde gerçekleşir. Bu yüzden FDA motorlara elektronik komütasyonlu motor da denmektedir ve firçalı motorlara iyi bir alternatif olarak görülmektedir (Xu, Li, \& Tang, 2008).

FDA motorlar kullanım amacına ve isteğe göre hız, konum ya da moment açısından kontrol edilebilirler. FDA motorlar farklı açılardan kontrol edilebildiği gibi farklı kontrolcülerle de kontrol edilebilirler. Birçok gelişmiş kontrol yöntemi olmasına rağmen, orant1-integral-türev (PID) kontrol; kararlılığı, karmaşık olmayan yapısı, geniş bir alana uygulanabilirliği ve dijital/analog platformlarda uygulama kolaylığı gibi nedenlerden dolayı hala endüstride en çok tercih edilen ve kullanılan kontrol yöntemidir (Anwar \& Pan, 2013).

PID kontrole sahip klasik bir sistemde PID yapısı ileri yönde beslemeli şekilde kullanılır. Fakat bazı çalışmalarda sistemin çıkış sinyalini iyileştirme amacıyla PID’deki orantı-integral-türev özelliklerinin sistemde yaptığı değişiklikler değerlendirilerek bunlar geri beslemede de kullanılmıştır.

Kumar ve arkadaşları değiştirilmiş PID (I-PD) yapısıyla FDA motorunun kontrolünü yapmış ve ileri beslemeli PID kontrolden daha başarılı sonuçlar elde etmişlerdir. Çalışmada ileri yönde I(integral) yapısı kullanılırken I'nın çıkışına geri besleme olarak PD yapısı eklenmiştir (Kumar, Swain, \& Neogi, 2017).

Kim ve arkadaşlarının yaptığı çalışmada ise ileri beslemeli PID yapısına geri beslemeli kontrol bileşenleri eklenerek klasik ileri yönlü beslemeli PID kontrol sistemine karşı üstünlükler ortaya konmuştur (Kim, Yang, Lim, \& Suh, 2010). Shekhar ve arkadaşları ise çalışmasında I-PD kontrolcünün ileri beslemeli PID’den daha iyi sonuçlar verdiğini teyit etmiş fakat ileri beslemeli PID kontrolcüsünün parametrelerini LQR (linear quadratic regulator) yardımıyla elde ederek I-PD gibi geliştirilmiş PID yapılarından daha üstün bir kontrolcü elde edilebileceğini ifade etmiştir. Böylece kontrolcü parametrelerinin belirlenmesinin de kontrolcü yapısı kadar performans açısından önemli olduğunu göstermiştir (Shekhar, Saha, \& Thakura, 2020).

PID kontrollerde, kontrolcü parametrelerinin belirlenmesinde en yaygın kullanılan yöntem Ziegler-Nichols metodu olsa da son yıllarda yapay zeka optimizasyon algoritmalarının da çokça kullanıldığı görülmektedir. Bunlardan (Ibrahim, Hassan, \& Shomer, 2014)'de parçacık sürü optimizasyonu (PSO), (Ansari, Alam, \& Jafri, 2011)'de genetik algoritma (GA), (Tarczewski \& Grzesiak, 2018)'de yapay arı kolonisi algoritması (YAKA) ve çiçek tozlaşma algoritması (ÇTA) PID kontrolcünün parametrelerinin belirlenmesinde kullanılmış ve hepsinde de başarılı sonuçlar elde edilmiştir.

Gün geçtikçe yeni yapay zeka optimizasyon algoritmaları keşfedilmekte ve mevcut problemler üzerinde denenmektedir (Tabak, Özkaymak, Tahir, \& Oktay, 2017). Kambur balinaların beslenme yöntemlerinden esinlenilerek ortaya çıkan ve başarılı uygulamaları olan Balina Optimizasyon Algoritması (BOA), son zamanlarda bulunan metasezgisel algoritmalardan bir tanesidir.

Literatürde BOA’nın uygulandığı çalışmalara bakıldığında, enerji kayıplarının azaltılması için bataryaların boyutlandırılması ve yerleşiminde (Ai, Ramachandaramurthy, Walker, \& Taylor, 2019), PV panellerin parametre tahmininde (Long, Wu, Jiao, Tang, \& Xu, 2020), elektrikli araç şarj istasyonlarının yeterli kapasite ile yerleştirilmesi probleminde (H. Zhang, Tang, Yang, \& Lan, 2019) ve yakıt pili terminal voltajı kontrolünde (Cao, Li, Zhang, Jermsittiparsert, \& Nasseri, 2020) kullanıldı̆̆ görülmektedir.

Bizim çalışmamızda ise FDA motorların hız kontrolünü gerçekleştirmek amacıyla ileri yönlü beslemeli PID’ye ek olarak geri beslemeli PD yapısı eklenerek işaretin maksimum aşma miktarının ve oturma zamanının düşürülmesi amaçlanmıştır. Yeni elde edilen PID/PD kontrolcüye sahip sistem ile ileri yönlü beslemeli PID kontrolcüye sahip sistem performans açısından karşılaştırılmıştır. Çalışmada kontrolcü parametrelerinin belirlenmesi için az sayıda parametre kullanması ve yerel optimum tuzaklarından kaçınması gibi üstünlüklerinden (Nasiri \& Khiyabani, 2018) dolayı BOA tercih edilmiştir. Bunun yanında parametreleri BOA vasıtasıyla elde edilen ileri yönlü PID beslemeli sistemin sonuçları literatürdeki benzer çalışmaların sonuçları ile karşılaştırılmıştır. Son olarak motorun hızı 1.5 katına çıkarılarak sistemin dinamik testi gerçekleştirilmiş ve PID ile PID/PD kontrolcüye sahip sistemler performans kriterleri açısından birbirleri ile karşılaştırılmıştır.

\section{Materyal ve Metot}

\subsection{Firçasız DA Motorlar ve Modellenmesi}

Fırçasız DA motorların sabit mekanik tork sağlaması (düzgün ikizkenar yamuk şeklindeki ters-e.m.k. ve stator faz komütasyonunun doğru uygulandığı zamanlar) ve yüksek mekanik güç yoğunluğuna sahip olması gibi nedenlerden dolayı kullanımı giderek yaygınlaşmaktadır (Siostrzonek \& Pirog, 2007). Şekil 1'de 3 fazlı bir FDA motorun elektrik eş değer devresi görülmektedir. 


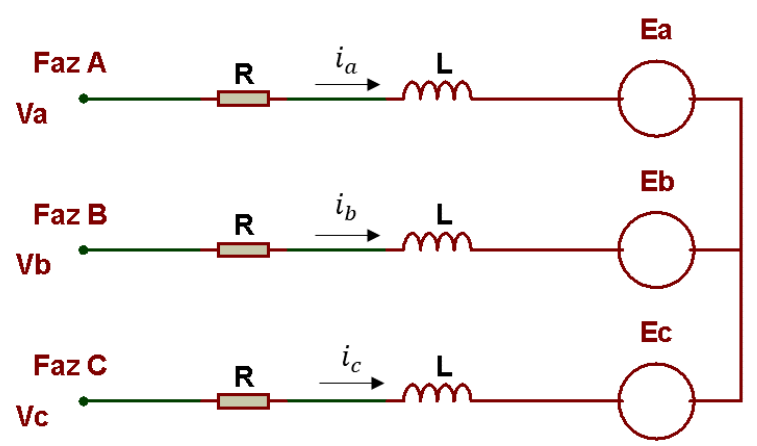

Şekil 1. FDA motorun elektrik eş değer devre ile gösterimi

Denklem 1-4 arasında FDA motorların temel eşitlikleri verilmiştir. Burada $V_{a}, i_{a}, R_{a}$ ve $L_{a}$ sırasıyla armatür gerilimi (V), akımı $(\mathrm{A})$, direnci $(\Omega)$ ve indüktansını $(\mathrm{H})$ temsil etmektedir. $E_{a}$ ters e.m.k. gerilimi (V), $T_{e m}$ elektromanyetik moment (N.m), $J$ rotor atalet momenti (kg.m²), $B$ motor sürtünme katsayısı (kg.m.s/rad), $w$ açısal hızı $(\mathrm{rad} / \mathrm{s})$ göstermektedir.

$$
\begin{aligned}
& V_{a}=i_{a} R_{a}+L_{a} \frac{d i_{a}}{d t}+E_{a} \\
& T_{e m}=J \frac{d w}{d t}+B w+T_{L} \\
& E_{a}=K_{e} \cdot w \\
& T_{e m}=K_{t} \cdot i_{a}
\end{aligned}
$$

Burada $K_{e}$ ters e.m.k. sabitini, $K_{t}$ motorun armatür sabitini ifade etmektedir. Bu iki değer motorun manyetik alan kuvveti ve bobin sargı sayısı gibi fiziksel özellikleri ile ilgilidir (Mohan, Undeland, \& Robbins, 2007). Tablo 1 simülasyon çalışmasında kullanılan motora ait parametreleri göstermektedir.

\section{Tablo1. Kullanilan Motorun Parametreleri}

\begin{tabular}{c|c}
\hline Parametre & Değer \\
\hline Armatür Direnci $\left(R_{a}\right)$ & $21.2 \Omega$ \\
\hline Armatür Endüktansı $\left(L_{a}\right)$ & $0.052 \quad \mathrm{H}$ \\
\hline Atalet Momenti $(J)$ & $1 \times 10^{-5} \mathrm{~kg} \cdot \mathrm{m}^{2}$ \\
\hline Ters e.m.k. sabiti $\left(K_{e}\right)$ & $0.1433 \quad \mathrm{~V} . \mathrm{s} / \mathrm{rad}$ \\
\hline Motor sürtünme katsayısı $(B)$ & $1 \times 10^{-4} \quad \mathrm{~kg} . \mathrm{m} . \mathrm{s} / \mathrm{rad}$ \\
\hline Armatür sabiti $\left(K_{t}\right)$ & $0.1433 \quad \mathrm{~kg} . \mathrm{m} / \mathrm{A}$ \\
\hline
\end{tabular}

Şekil 2'de FDA motorun blok diyagramı verilmiştir. Burada $V_{t}(s)$ giriş işaretini, $w_{m}(s)$ motor açısal hızını göstermektedir.

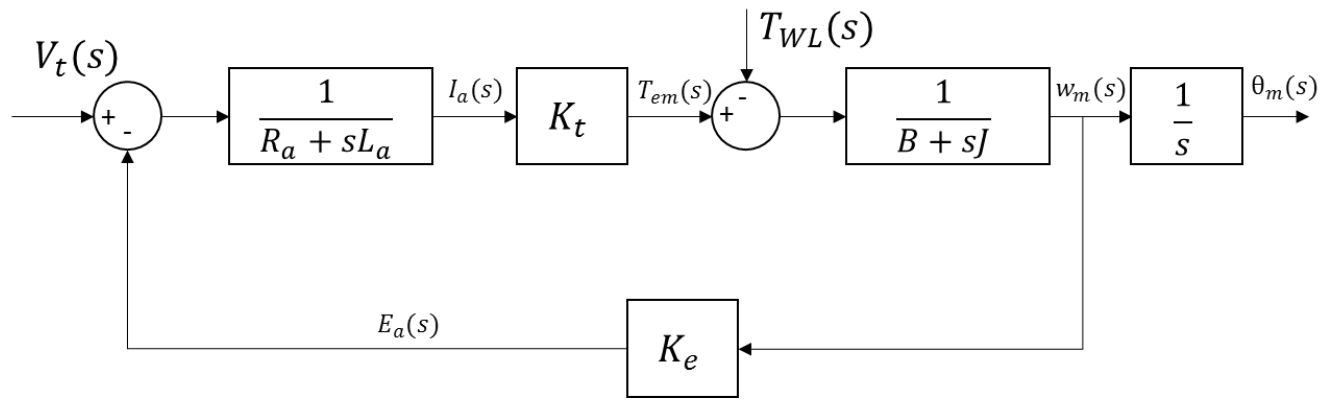

Şekil 2. FDA motor ve yükün blok diyagramı

Denklem 5'te FDA motorun transfer fonksiyonu verilmiştir.

$G(s)=\frac{W(s)}{V_{t}(s)}=\frac{K_{t}}{J \cdot L \cdot s^{2}+(J \cdot R+L \cdot B) \cdot s+\left(B \cdot R+K_{e} \cdot K_{t}\right)}$

\subsection{Sistemin Kontrolü}

Çalışmada FDA motorun hız kontrolünü gerçekleştirebilmek için PID ve PID/PD kontrolcüler kullanılmıştır. 


\subsubsection{PID Kontrol}

PID kontrol en yaygın kullanılan kontrolcü çeşididir. Şekil 3’te PID kontrolün kullanıldığı klasik bir sistemin blok diyagramı gösterilmektedir. Burada $r$ referans girişini, $e$ giriş ile sistem geri beslemenin farkı olan hatayı, $u$ kontrolcü çıkşıını, $y$ ise sistemin çıkışını ifade etmektedir.

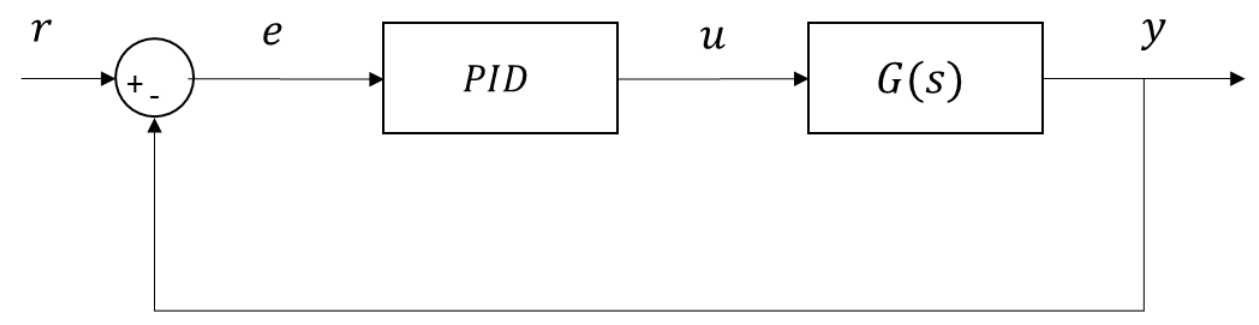

Şekil 3. PID kontrolün kullanıldı̆ğ klasik bir sistemin blok diyagramı ile gösterimi

Denklem 6'da PID kontrolcünün parametrelerini içeren transfer fonksiyonu verilmiştir. PID içerisindeki $K_{p}, K_{i}$ ve $K_{d}$ parametreleri en uygun değerleri alarak sistem çıkışı ile referans girişi arasındaki farkı ifade eden hata değerini sıfira indirmiş olur.

$$
C(s)=K_{p}+\frac{K_{i}}{s}+K_{d} s
$$

PID kontrol yapısının kullanıldığı klasik bir sistemin girişi ve çıkışı arasındaki kapalı döngü transfer fonksiyonu Denklem 7'de verilmiştir. Burada $G(s)$ kontrol edilecek sistemin, $C(s)$ ise kontrolcünün transfer fonksiyonunu ifade etmektedir.

$$
\frac{y}{r}=\frac{G(s) C(s)}{1+G(s) C(s)}
$$

\subsubsection{PID/PD Kontrol}

PID kontrolde $K_{p}$, $K_{i}$ ve $K_{d}$ parametrelerinin her biri sistemin çıkışını farklı etkilemektedir. Örneğin $K_{p}$ ve $K_{i}$ parametreleri maksimum aşmayı artıırken $K_{d}$ parametresi maksimum aşmayı azaltmaktadır. $K_{p}$ ve $K_{i}$ parametreleri yükselme zamanını düşürür. Oturma zamanına etkisi açısından değerlendirildiğinde $\mathrm{K}_{\mathrm{i}}$ bu zamanı artırırken $\mathrm{K}_{\mathrm{d}}$ azaltır (Syed Hussien, Jaafar, Ghazali, \& Abdul Razif, 2015).

Bu bilgiler eşliğinde sistemin dizaynı değiştirilebilir. Çalışmada kullanılan geliştirilmiş PID (PID/PD) kontrol yapısı Şekil 4'te gösterilmiştir. Burada ileri yönde PID kontrolcü kullanılırken geri yönde PD kontrolcü ile PID çıkışına negatif geri besleme yapılmıştır. Bu geri beslemeli sistemdeki asıl amaç ilk etapta oluşan maksimum aşma miktarını ve oturma zamanını en aza indirerek klasik PID kontrol yapısına göre sistemde iyileştirme yapmaktır.

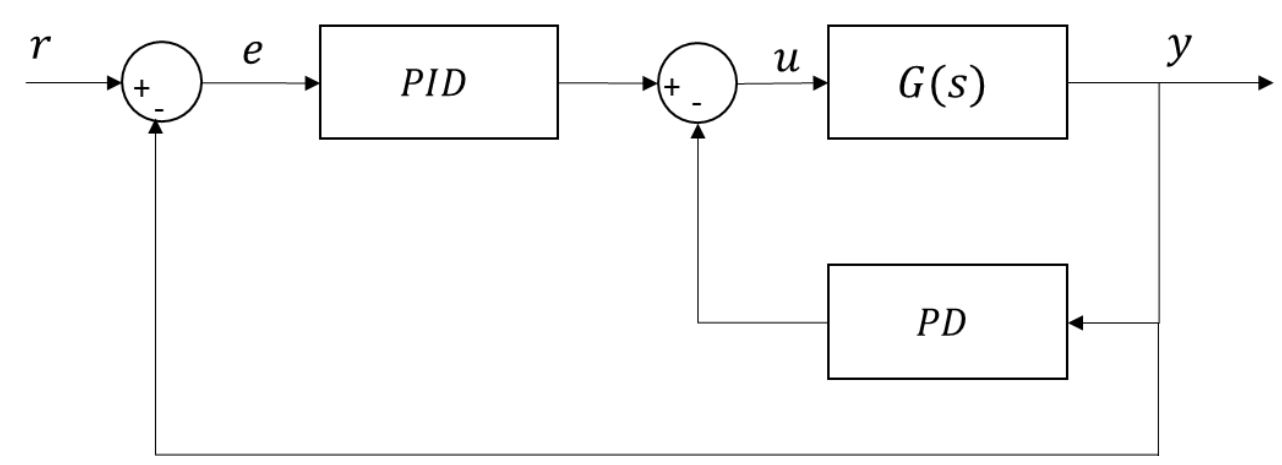

Şekil 4. Gelişstirilmiş PID (PID/PD) kontrol yapısının blok diyagramı ile gösterimi

Denklem 8'de geliştirilmiş PID (PID/PD) yapısının kullanıldığı sistemin girişi-çıkışı arasındaki transfer fonksiyonu verilmiştir.

$$
\frac{y}{r}=\frac{G(s) C_{1}(s)}{1+G(s)\left(C_{1}(s)+C_{2}(s)\right)}
$$

Burada $G(s)$ kontrol edilecek sistemin transfer fonksiyonunu, $C_{1}(s)$ ve $C_{2}(s)$ sirasiyla kontrolcülerin transfer fonksiyonunu göstermektedir ve Denklem 9 ile 10 'da verilmiş̧tir.

$$
\begin{aligned}
& C_{1}(s)=K_{p 1}+\frac{K_{i 1}}{s}+K_{d 1} s \\
& C_{2}(s)=K_{p 2}+K_{d 2} s
\end{aligned}
$$

Denklem 9 ve 10'da iki farklı kontrolcü yapısı olduğu için ifadelerde 1-2 rakamları kullanılmıştır. 


\subsubsection{Kontrolcü Parametrelerinin Belirlenmesi}

Kontrolcü parametrelerinin belirlenmesi de kontrolcü yapısının olușturulması kadar önemlidir. PID kontrolde parametrelerin belirlenmesi için Cohen-Coon yöntemi, Tyreus-Luyben yöntemi ve Ziegler-Nichols yöntemi gibi birtakım temel yöntemler mevcuttur. Bunlardan Ziegler-Nichols yöntemi halen en çok kullanılan ayar yöntemlerinden birisidir (Uysal, 2010). Ancak yapılan çalışmalara bakıldığında geleneksel parametre ayarlama yöntemleri; yüksek aşma miktarı, düşük doğruluk oranı ve ayar süresinin uzun olması gibi dezavantajlarından dolayı yapay zeka optimizasyon algoritmaları kadar başarılı olamamaktadır (Y. Zhang, Zhang, \& Dong, 2019). Çalışmada yapay zeka optimizasyon algoritmalarından olan BOA yöntemi kontrolcü parametrelerinin belirlenmesi için kullanılmışır.

\subsubsection{Balina Optimizasyon Algoritması}

Balina optimizasyon algoritması, Mirjalili ve Lewis tarafindan 2016 yılında, kambur balinaların yaptı̆g ve hava kabarcığı yöntemi olarak tabir edilen bir avlanma yönteminden esinlenerek ortaya atılmıştır. Yedi farklı balina türünden biri olan kambur balinalar genellikle kriller ve yüzeye yakın balıklarla beslenmektedirler. Avlanma esnasında balinalar avın etrafinda bir daire veya "9" şeklinde spiral çizerek kabarcık oluştururlar. Bu kabarcık sayesinde avdan kendini gizleyebilir, avın dikkatini dağıtabilir ve avlanma işlemini kolaylaştırabilirler. Kabarcıkların oluşumundan sonra kambur balinalar yüzeye doğru hızlıca yüzerek avlarını elde ederler (Goldbogen et al., 2013). Şekil 5 kambur balinaların hava kabarcığı oluşturarak avlanmasını göstermektedir. BOA'da avlanma stratejisi; avın etrafını sarma, ava saldırı ve avı arama olmak üzere 3 kısımda modellenmiştir.

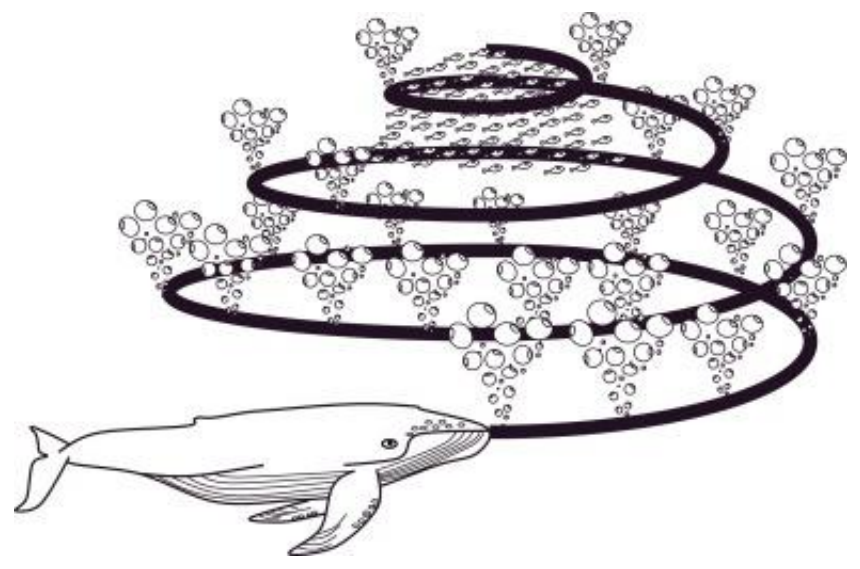

Şekil 5. Kambur balinaların hava kabarcığı yöntemi ile beslenme davranışı

\subsubsection{Avın Etrafinı Sarma}

Kambur balinalar avlarının yerlerini tahmin ederek onların çevresini hava kabarcıkları ile sararlar. BOA'da av, ulaşılması mümkün optimum nokta olarak kabul edilir. Öncelikle en iyi arama aracı belirlenir, ardından diğer arama araçlarının pozisyonu en iyi arama aracına göre güncellenir. Bu hareketin matematiksel modeli Denklem 11 ve 12'deki gibidir (Mirjalili \& Lewis, 2016).

$$
\begin{aligned}
& \vec{D}=\left|\vec{C} \cdot \overrightarrow{X^{*}}(t)-\vec{X}(t)\right| \\
& \vec{X}(t+1)=\overrightarrow{X^{*}}(t)-\vec{A} \cdot \vec{D}
\end{aligned}
$$

Buradaki 't' o anki iterasyonu $\vec{A}$ ve $\vec{C}$ katsayılar vektörünü, $\vec{X}$ pozisyon vektörünü, $X^{*}$ karşılaşılan en iyi çözüm vektörünü, '.' matris çarpımını göstermektedir. Eğer burada daha iyi bir çözüm elde edilirse $X^{*}$ güncellenir. $\vec{A}$ ve $\vec{C}$ katsayı vektörlerinin hesaplanması için Denklem 13 ve 14 'ten faydalanılır.

$$
\begin{aligned}
\vec{A} & =2 \vec{a} \cdot \vec{r}-\vec{a} \\
\vec{C} & =2 \cdot \vec{r}
\end{aligned}
$$

Burada $\vec{a}$, her iterasyonda 2'den başlayıp 0'a doğru lineer olarak azalan bir vektör, $\vec{r}$ ise $[0,1]$ aralığında rastgele değişen bir vektörü ifade etmektedir.

\subsubsection{Ava Doğru Hareket Etme}

Bu davranış avın etrafındaki çemberi daraltma ve spiral oluşturma hareketi olarak iki aşamada modellenmiştir. Denklem 13 'teki $\vec{a}$ değeri azaltılarak avın etrafındaki çemberin daralması sağlanır. Spiral oluşturma hareketinde ise hedef konum ile arama aracı arasındaki mesafe hesaplanarak Denklem 15 elde edilir. 


$$
\vec{X}(t+1)=\overrightarrow{D^{\prime}} \cdot e^{b l} \cdot \cos (2 \pi l)+\overrightarrow{X^{*}}(t)
$$

Denklem $15^{\prime}$ te arama aracı ile elde edilen en iyi nokta arasındaki mesafeyi veren $\overrightarrow{D^{\prime}}=\left|\overrightarrow{X^{*}}(t)-\vec{X}(t)\right|$ formülünden faydalanılır. Ayrıca Denklem 15'teki $b$ spiralin şeklinin belirlenmesinde kullanılan logaritmik sabit, $l[-1,1]$ arasında rastgele değişen bir değerdir. Kambur balinalar yüzeye yakın noktalardaki avına doğru yüzerken aynı zamanda spiral oluşturma hareketini yaparlar. Spiral oluşturma şeklinde ya da doğrusal şekilde hareketten hangisinin yapılacağına ise Denklem 16'da verildiği gibi olasılık üzerinden karar verilir. İki hareketten hangisinin seçileceği 0.5 olasılıkla tanımlanmıştır.

$$
\vec{X}(t+1)=\left\{\begin{array}{cc}
\overrightarrow{X^{*}}(t)-\vec{A} \cdot \vec{D} & , p<0.5 \\
\overrightarrow{D^{\prime}} \cdot e^{b l} \cdot \cos (2 \pi l)+\overrightarrow{X^{*}}(t) & , p>0.5
\end{array}\right.
$$

Denklem 16'daki $p$ değeri $[0,1]$ arasında değişen rastgele bir değerdir.

\subsubsection{Av Arama}

Global arama için arama araçlarının yeni konumlarının belirlenmesi, elde edilen en iyi noktaya göre değil rastgele seçilen arama aracı etrafında yapılır. Bu durum Denklem 17 ve 18 'de verilmiştir.

$$
\begin{aligned}
& \vec{D}=\left|\vec{C} \cdot \vec{X}_{\text {rand }}-\vec{X}\right| \\
& \vec{X}(t+1)=\vec{X}_{\text {rand }}-\vec{A} \cdot \vec{D}
\end{aligned}
$$

Burada $\vec{X}_{\text {rand }}$, rastgele seçilen arama aracını göstermektedir. Global veya yerel aramalar arasındaki seçim ise $\vec{A}^{\prime}$ nın değerine göre belirlenir. Burada $\vec{A}>1$ ve $\vec{A}<-1$ olduğu durumlarda arama global arama olarak algılanır ve Denklem 17 ve 18 'de uygulanır (Mirjalili \& Lewis, 2016).

\section{Analiz ve Simülasyon Sonuçları}

Kontrolcü parametrelerinin belirlenmesinde yapay zeka optimizasyon algoritmalarının kullanılabilmesi için bir amaç fonksiyonuna ihtiyaç duyulmaktadır. Aslında optimizasyon algoritması bir fonksiyonun değerini minimuma indirirken o fonksiyonu oluşturan değişkenlerin değerlerini optimum değere taşır. O yüzden minimum yapılacak fonksiyona amaç fonksiyonu denir (Tabak, Kayabasi, Guneser, \& Ozkaymak, 2019). PID kontrolde kullanılmak üzere farklı amaç fonksiyonları mevcuttur. Bu amaç fonksiyonlarından en çok tercih edilenleri; hata karelerinin toplamı (ISE), mutlak hataların toplamı (IAE), zaman ağılıklı hata karelerinin toplamı (ITSE), zaman ağılıklı mutlak hataların toplamı (ITAE) şeklindedir (Hajísalm \& Altaş, 2014). Bizim çalışmamızda hata karelerinin toplamı şeklindeki amaç fonksiyonundan faydalanılmıştır. BOA yönteminde popülasyon sayısı 50 , iterasyon sayısı da 250 olarak alınmış ve çalışma MATLAB 2016a programında simüle edilmiş̧ir.

Çalışmada ilk olarak PID kontrolcünün kullanıldığı sistemin optimizasyonu yapılarak sistemin cevabı literatürde daha önce yapılan çalışmaların sonuçları ile karşılaştııılmıştır. Ardından PID/PD yapısı kullanılarak elde edilen sistem BOA ile optimize edilmiştir. PID/PD yapısının kullanıldığı sistem ile PID kontrolcünün kullanıldığı sistem performans açısından karşılaştırılmıştır. Tablo 2'de FDA motorun kontrolünde farklı algoritmalarla elde edilen PID/PD ve PID kontrolcülerin parametreleri gösterilmiştir. BOA-PID/PD ve BOA-PID çalışması literatürde yapılan GA-PID, PSO-PID, LQ-PID ve LQR-PID yöntemleri ile karşılaştırılmıştır. $\mathrm{K}_{\mathrm{p} 2}$ ve $\mathrm{K}_{\mathrm{d} 2}$ değerleri BOA-PID/PD çalışmasındaki PD parametrelerini temsil etmektedir.

\section{Tablo 2. Algoritmalara göre kontrolcü parametrelerinin değerleri}

\begin{tabular}{c|c|c|c|c|c|c}
\hline Parametreler & BOA-PID/PD & BOA-PID & $\begin{array}{c}\text { LQ-PID (Kim et } \\
\text { al., 2010) }\end{array}$ & $\begin{array}{c}\text { GA-PID (Kim } \\
\text { et al., 2010) }\end{array}$ & $\begin{array}{c}\text { PSO-PID } \\
\text { (Kim et al., } \\
\text { 2010) }\end{array}$ & $\begin{array}{c}\text { LQR-PID (Yu \& } \\
\text { Hwang, 2004) } \\
\text { (Kim et al., 2010) }\end{array}$ \\
\hline $\mathrm{K}_{\mathrm{p}}$ & 3.2009 & 124.6917 & 72.423 & 93.162 & 190.018 & 70.556 \\
\hline $\mathrm{K}_{\mathrm{i}}$ & 0.1998 & 33.3382 & 14.515 & 38.623 & 50 & 10 \\
\hline $\mathrm{K}_{\mathrm{d}}$ & 0.7978 & 0.0436 & 0.0311 & 0.0278 & 0.0396 & 0.022 \\
\hline $\mathrm{K}_{\mathrm{p} 2}$ & 1.00018 & - & - & - & - & - \\
\hline $\mathrm{K}_{\mathrm{d} 2}$ & 20.0184 & - & - & - & - & - \\
\hline
\end{tabular}

Tablo 3’te optimizasyonda farklı algoritmaların kullanılması sonucu elde dilen performans çıktıları görülmektedir. Buna göre BOA-PID/PD \%0.05524'lük maksimum aşma ve 0.36007 ms'lik oturma zamanı açısından PID kontrolcülerden çok daha iyi performans göstermiştir. Yükselme zamanı açısından karşılaştırıldığında ise diğer yöntemlerin çok az gerisinde kalmaktadır fakat 3 kriter birlikte değerlendirildiğinde BOA-PID/PD’nin üstünlüğünden söz etmek mümkündür. Diğer yandan PID kontrolcülerin kendi içindeki karşılaştırmaya bakacak olursak BOA-PID \%10.685'lik maksimum aşma ile en iyi değere sahiptir. Bunun yanında yükselme zamanı ve oturma zamanı açısından PSO-PID’nin az bir farkla gerisinde kalmaktadır. Fakat PSO-PID'deki maksimum aşmanın \%16.85 olduğu göz önünde bulundurulursa BOA-PID’nin üstünlüğünden söz edilebilir. BOA-PID’nin diğer bir karşılaştırması da LQPID ile yapılabilir. Çünkü LQ-PID maksimum aşma açısından BOA-PID’nin hemen arkasından ikinci sırada gelmektedir. Yükselme zamanı ve oturma zamanı açısından da değerlendirilecek olunursa BOA-PID, LQ-PID'den her alanda daha iyi performans göstermiş diyebiliriz. Son olarak tabloya bakarak BOA-PID’nin üç kriter açısından da GA-PID ve LQR-PID'den üstün performans gösterdiğini söyleyebiliriz. 
European Journal of Science and Technology

Tablo 3. Algoritmaların performans karşılaştırmaları

\begin{tabular}{l|c|c|c}
\hline Yöntem & Maksimum Așma (\%) & Yükselme Zamanı (ms) & Oturma Zamanı (ms) \\
\hline BOA-PID/PD & 0.05524 & 0.21108 & 0.36007 \\
\hline BOA-PID & 10.685 & 0.128212 & 0.91363 \\
\hline GA-PID (Kim et al., 2010) & 15.878 & 0.171 & 1.006 \\
\hline PSO- PID (Kim et al., 2010) & 16.85 & 0.118 & 0.70 \\
\hline LQ-PID (Kim et al., 2010) & 10.98 & 0.176 & 1.167 \\
\hline LQR-PID (Yu \& Hwang, & 17.545 & 0.204 & 1.146 \\
2004)(Kim et al., 2010) & & & \\
\hline
\end{tabular}

Şekil 6'da algoritmalara göre PID kontrolcüye sahip sistemlerin performans karşılaştırmaları gösterilmiştir. Daha doğru bir karşılaştırma yapabilmek için literatürde aynı parametrelere sahip motorlar üzerinde yapılmış çalışmalar göz önünde bulundurulmuştur ve referans hız $1000 \mathrm{rpm}$ olarak alınmıştır. Farklı algoritmalarla optimize edilen sistemin çıkışlarına bakıldığında maksimum aşmanın \%10 seviyesinin üzerinde olduğu ve içlerinde en düşüğünün \%10.685 ile BOA-PID’nin olduğu görülmektedir. Yine şekilden de anlaşılabileceği gibi tüm performans kriterleri beraber düşünüldüğünde BOA-PID diğer yöntemlere karşı daha iyi durumdadır.

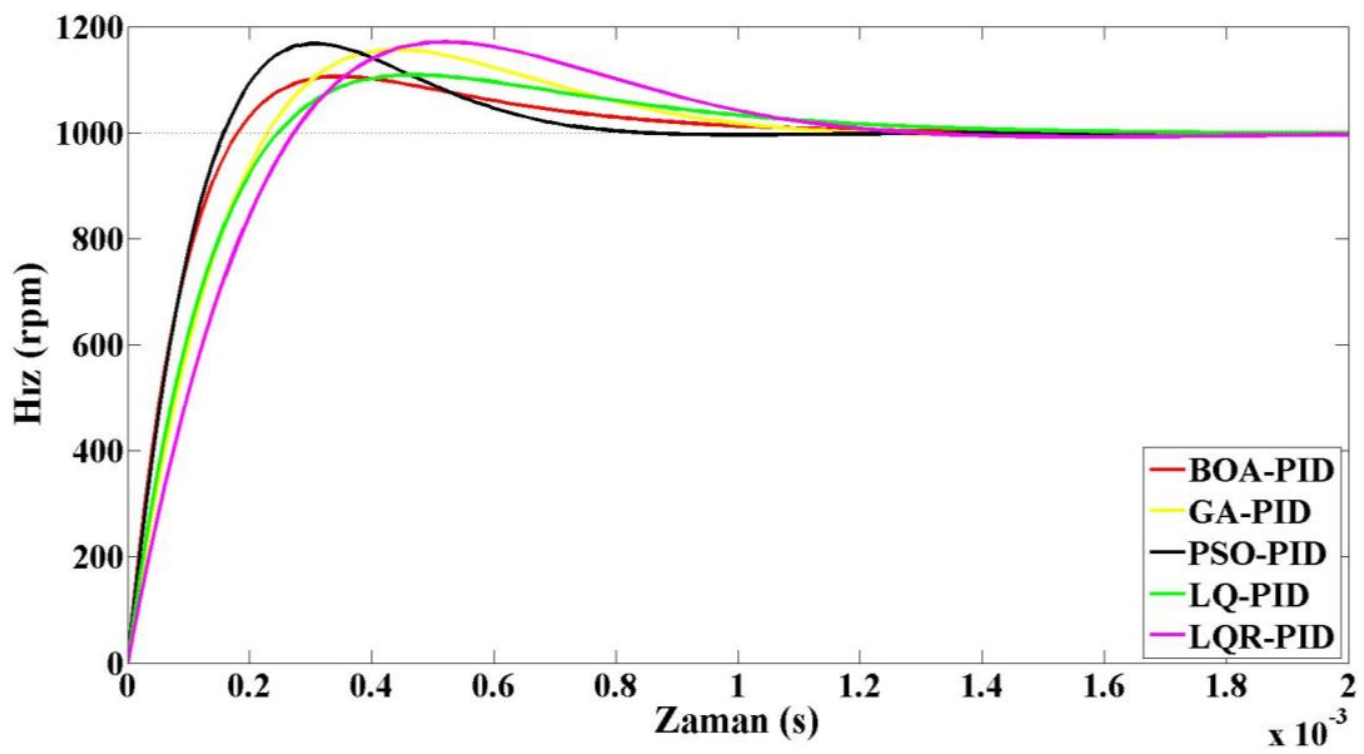

Şekil 6. PID kontrolde farklı algoritmaların performans karşılaştırması

Şekil 7'de BOA kullanılarak parametreleri belirlenen PID ile PID/PD'nin performanslarının karşılaştırması görülmektedir. Şekilden de görüldüğü gibi PID/PD kontrol yapısının kullanıldığı sistem PID kontrolcünün kullanıldığı sisteme göre maksimum aşma ve oturma zamanı açısından daha üstün performans sergilemiş̧tir. PID kontrolcüye sahip sistem yükselme zamanı açısından 0.08287 ms'lik farkla daha iyi performans göstermiş durumdadır. PID/PD kontrol yapısının kullanıldığı sistemde az miktarda yükselme zamanı artışına karşılık maksimum aşma ve oturma zamanında çok daha fazla iyileşme gözlenmiştir. Performans karşılaştırmasına sayısal olarak bakıldığında ise BOA-PID/PD'nin BOA-PID'den oturma zamanı açısından $0.55356 \mathrm{~ms}$ daha hızlı, maksimum aşma açısından da yaklaşı 193 kat daha iyi olduğu görülmektedir. 


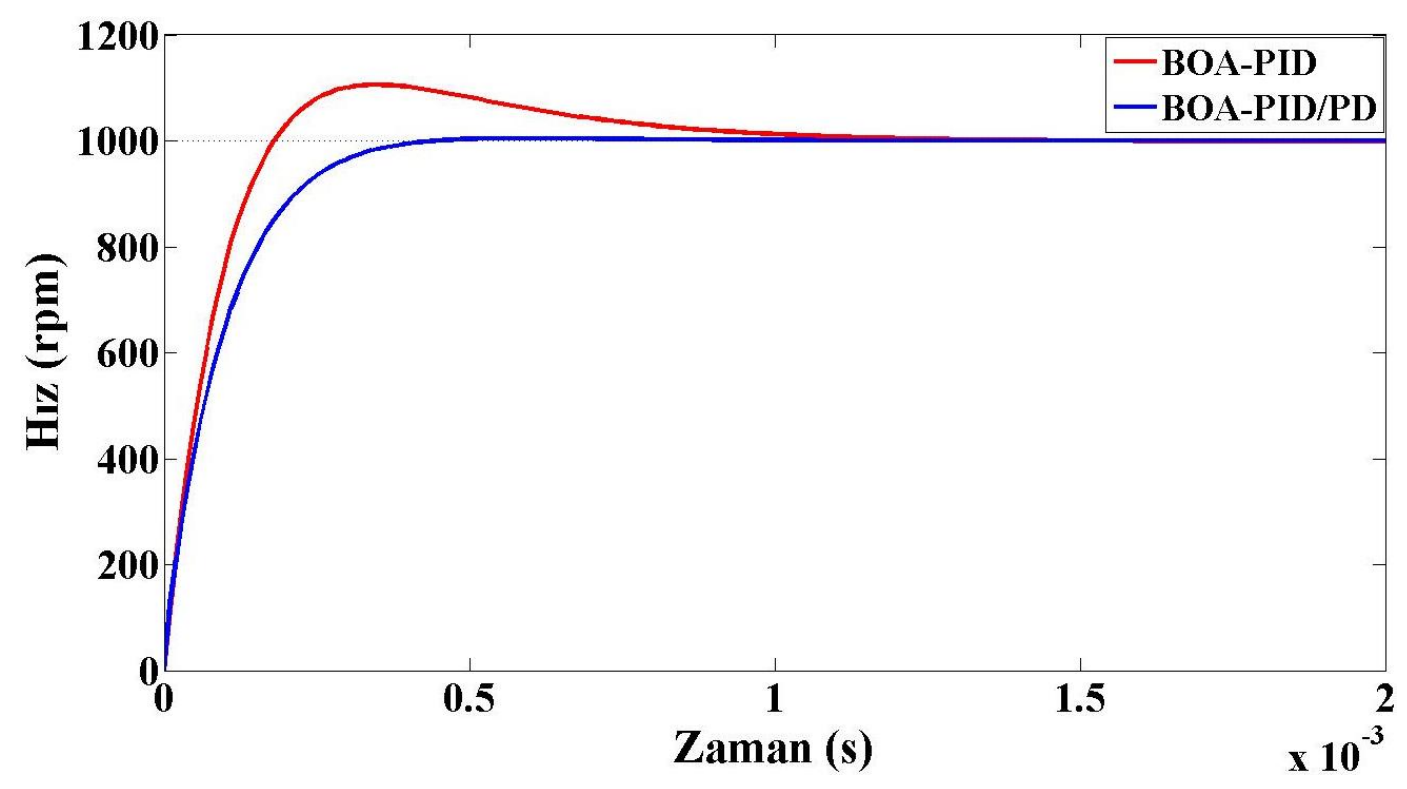

Şekil 7. PID-PID/PD kontrolcülerin performans karşılaştırması

FDA motorun dinamik testinin yapılması amacıyla 1000 rpm hızında çalışan motorun hızı aniden 1500 rpm'e çıkarılmıştır. Tablo 4 'te hızı 1.5 kat artırılan sistemin algoritmalara göre performans çıktıları verilmiştir. Tablodan da anlaşılacağı gibi ilk kalkış anında BOAPID/PD'deki \%0.05524'lük maksimum aşmanın \%0'a düştüğü, BOA-PID’deki maksimum aşmanın üçte birine düştüğü ve diğer yöntemlerle elde edilen maksimum aşmaların \%3-5'ler seviyesine düştüğü görülmektedir. BOA-PID/PD’nin FDA motordaki hızlanmaya karşın maksimum aşmayı sıfıra indirmesi ve oturma zamanında ciddi düşüş sergilemesi diğer sistemlere olan üstünlüğünü ortaya koymaktadır. Diğer yandan FDA motordaki hız değişimine karşı BOA-PID, PSO-PID’den maksimum aşma göz önünde bulundurulduğunda 1.572 kat daha iyi performans gösterirken yükselme zamanı ve oturma zamanı açısından az da olsa PSO-PID’nin arkasındadır. Fakat 3 kriter de dikkate alındığında BOA-PID’nin PSO-PID’den az bir yükselme ve oturma zamanı karşılığında yüksek maksimum aşma farkı oluşturmasından dolayı tercih sebebi olacağı söylenebilir. BOA-PID’yi hız değişimi sonrasında maksimum aşma, oturma zamanı ve yükselme zamanı açısından GA-PID, LQ-PID ve LQR-PID ile karşılaştırılacak olunursa hepsinden daha iyi performans gösterdiği söylenebilir.

Tablo 4. Hız değişimi sonrasında algoritmaların performans karşılaştırmaları

\begin{tabular}{l|c|c|c}
\hline Yöntem & Maksimum Așma (\%) & Yükselme Zamanı (ms) & Oturma Zamanı (ms) \\
\hline BOA-PID/PD & 0 & 0.1808 & 0.4910 \\
\hline BOA-PID & 3.571 & 0.07036 & 0.6195 \\
\hline GA-PID (Kim et al., 2010) & 5.2790 & 0.100158 & 0.81114 \\
\hline PSO- PID (Kim et al., 2010) & 5.6139 & 0.06887 & 0.5759 \\
\hline LQ-PID (Kim et al., 2010) & 3.70688 & 0.097947 & 0.826619 \\
\hline LQR-PID (Yu \& Hwang, & 5.7845 & 0.12255 & 0.948599 \\
2004)(Kim et al., 2010) & & & \\
\hline
\end{tabular}

Şekil 8, PID kontrolcünün kullanıldığı sistemde hızın t=2 ms'de, 1000 rpm'den 1500 rpm'e çıkarılması sonucunda çıkışın algoritmalara göre cevaplarını göstermektedir. BOA-PID ilk kalkışta olduğu gibi hızın 1.5 katına çıktığı durumda da maksimum aşma açısından diğer algoritmalardan daha iyi sonuç vermiştir. 


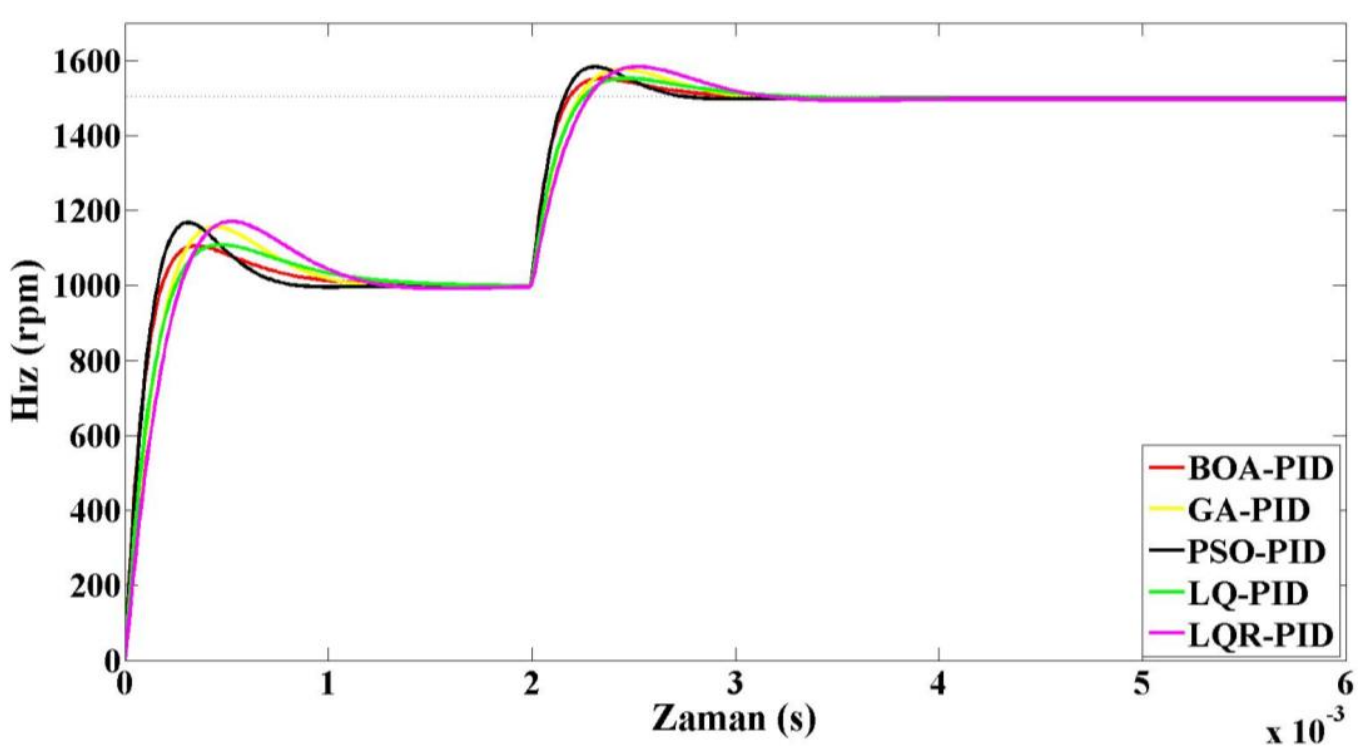

Şekil 8. PID kontrolde hız değişimi sonrası farklı algoritmaların performans karşılaştırması

Şekil 9'da FDA motorunun hızının 1.5 katına çıkarılması sonucunda BOA-PID/PD ve BOA-PID'nin karşılaştırması görülmektedir. Grafikten de anlaşılabildiği gibi PID/PD kontrol yapısının kullanıldığı sistemde hız artışına karşın aşma miktarı $\%$ 'dır. Bu haliyle BOA-PID/PD, BOA-PID'den hem maksimum aşma hem de oturma zamanı açısından daha yüksek performans göstermiştir. Yükselme zamanı açısından BOA-PID bir miktar daha iyi görünse de tüm kriterler değerlendirildiğinde BOAPID/PD’nin daha üstün olduğu açıkça görülmektedir.

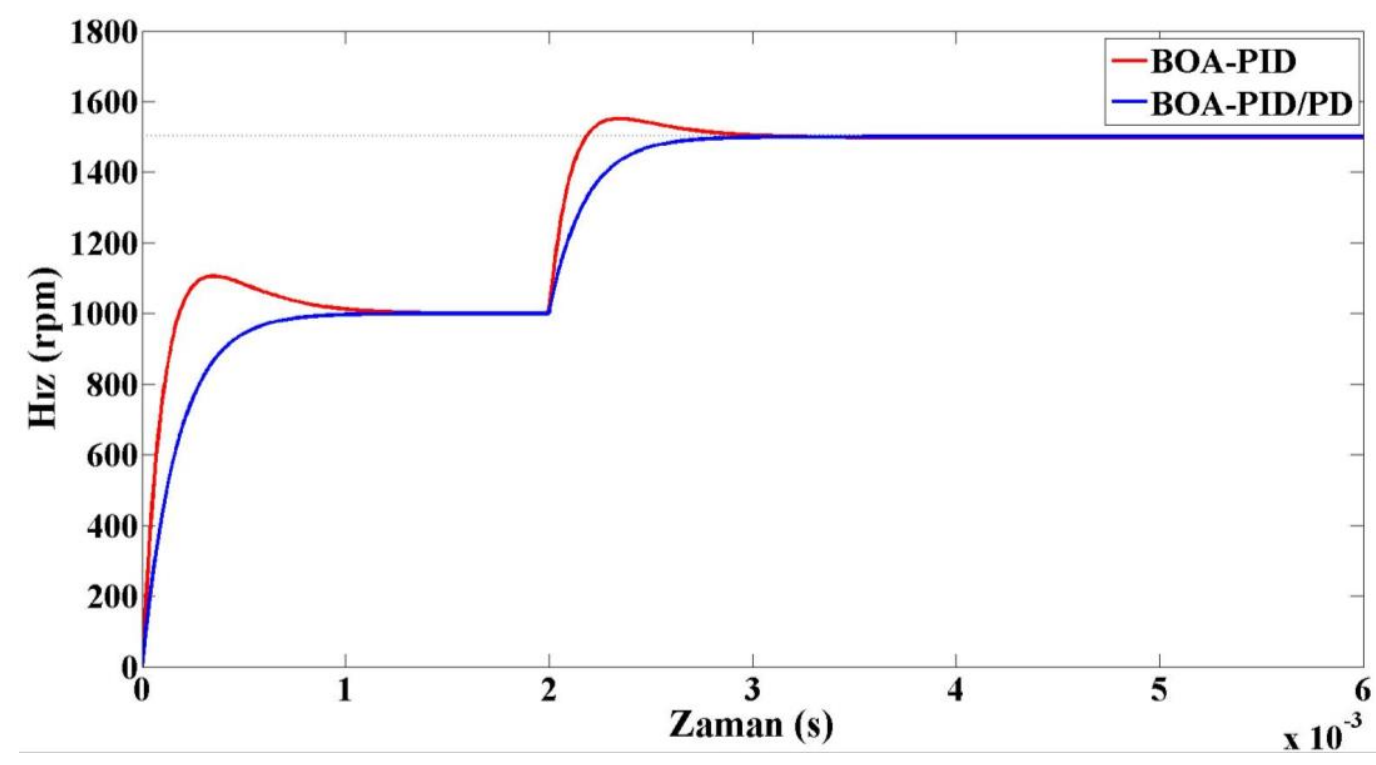

Şekil 9. PID-PID/PD kontrolcülerin hız değişimi sonrası performans karşılaştırması

\section{Sonuç}

Yapılan çalışmada, FDA motorunun hız kontrolü hem PID hem de PID/PD kontrolcü ile gerçekleştirilmiş olup maksimum aşma, oturma zamanı ve yükselme zamanı gibi kriterler açısından performansları karşılaştırılmıştır. Kontrol performansının artırılmasında kontrolcü yapısının önemi kadar parametre belirleme yönteminin de önemli olmasından dolayı son yıllarda bulunan ve farklı uygulamalarda başarılı sonuçlar veren BOA kullanılmıştır.

BOA ile elde edilen PID kontrolcünün sonuçları daha önce literatürde aynı özelliklere sahip FDA motorun hız kontrolünde kullanılan ve GA, PSO, LQ ve LQR metotları ile optimize edilen PID kontrolleri ile karşılaştırılmış, maksimum aşma konusunda diğer yöntemlerden daha iyi sonuç verdiği görülmüştür. Oturma zamanı ve yükselme zamanı açısından PSO-PID, BOA-PID’den çok az farkla daha iyi olsa da maksimum aşma oranı BOA-PID’nin 1.58 katıdır ve diğer tüm yöntemler içerisinde maksimum aşma oranı en yüksek olanıdır. Ayrıca BOA-PID üç kriter açısından da incelendiğinde GA-PID, LQ-PID ve LQR-PID'den daha iyi performans sergilediği görülmüştür. PID kontrolcülerin kullanıldığı çalışmaların içinde kriterlerin üçü de değerlendirildiğinde BOA-PID tercih edilmelidir. 
PID/PD kontrolcünün kullanıldığı sistem, çalışmanın asıl amacı olan maksimum aşmayı minimuma indirme ve oturma zamanını kısaltma gibi kriterler açısından bakıldığında PID kontrolcünün kullanıldığı sistemlerden çok daha üstün performans göstermiştir. Şöyle ki PID kontrolcülerin kullanıldığı sistemlerin içerisinde maksimum aşmanın en iyi olduğu yöntem \%10.685'lik oran ile BOAPID iken BOA-PID/PD'de bu değer \%0.05524'tür. Yine aynı şekilde PID kontrolcülerin kullanıldığı sistemlerin içerisinde en düşük oturma zamanına sahip olan yöntem 0.70 ms'lik zaman ile PSO-PID iken BOA-PID/PD'de bu değer 0.36007 ms'dir. Yükselme zamanı açısından incelendiğinde PID/PD kontrolcü yapısına sahip sistem PID kontrolcülü sistemlerin hemen arkasında olduğu fakat üç kriter birlikte değerlendirildiğinde PID/PD’nin PID’ye karşı baskın bir üstünlüğe sahip olduğu görülmektedir.

Ayrıca dinamik testi gerçekleştirmek amacıyla FDA motorun hızı 1.5 katına çıkarılarak hem PID ile PID/PD kontrolcüler karşılaştırılmış hem de PID kontrolcünün farklı optimizasyon algoritmaları ile elde edilen sonuçları karşılaştırılmıştır. Buradan elde edilen sonuç ise BOA-PID'nin diğer PID kontrolcülere göre daha iyi performans sergilemiş olmasıdır. PID/PD kontrolcü, maksimum aşmayı \%0'a ve oturma zamanını 0.4910 ms'ye çekerek PID’ye karşı olan üstünlüğünü göstermiştir. Daha sonraki çalışmalarda geliştirilmiş PID yapıları, FDA motorların tork kontrolü gibi uygulamalarında kullanılabilir. Bunu gerçekleştirirken de farklı, yeni ve güçlü yapay zeka optimizasyon algoritmalarından faydalanılabilir.

\section{Kaynakça}

Ai, L., Ramachandaramurthy, V. K., Walker, S. L., \& Taylor, P. (2019). Optimal placement and sizing of battery energy storage system for losses reduction using whale optimization algorithm. Journal of Energy Storage, 26(May), 100892.

https://doi.org/10.1016/j.est.2019.100892

Ansari, U., Alam, S., \& Jafri, S. M. U. N. (2011). Modeling and control of three phase BLDC motor using PID with genetic algorithm. Proceedings - 2011 UKSim 13th International Conference on Modelling and Simulation, UKSim 2011, 189-194. https://doi.org/10.1109/UKSIM.2011.44

Anwar, M. N., \& Pan, S. (2013). Synthesis of the PID controller using desired closed-loop response. In IFAC Proceedings Volumes (IFAC-PapersOnline) (Vol. 10). https://doi.org/10.3182/20131218-3-IN-2045.00023

Cao, Y., Li, Y., Zhang, G., Jermsittiparsert, K., \& Nasseri, M. (2020). An efficient terminal voltage control for PEMFC based on an improved version of whale optimization algorithm. Energy Reports, 6, 530-542. https://doi.org/10.1016/j.egyr.2020.02.035

Goldbogen, J. A., Friedlaender, A. S., Calambokidis, J., McKenna, M. F., Simon, M., \& Nowacek, D. P. (2013). Integrative Approaches to the Study of Baleen Whale Diving Behavior, Feeding Performance, and Foraging Ecology. BioScience, 63(2), 90100. https://doi.org/10.1525/bio.2013.63.2.5

GRIGORIE, T. L., KHAN, S., BOTEZ, R. M., MAMOU, M., \& MÉBARKI, Y. (2019). Design and experimental testing of a control system for a morphing wing model actuated with miniature BLDC motors. Chinese Journal of Aeronautics. https://doi.org/10.1016/j.cja.2019.08.007

Hajİsalm, A., \& Altaş, İ. H. (2014). Hibrit Rüzgar / F V Enerji Sistemleri Iç̧in P ID Denetleyici Parametrelerinin PSO ve GA ile Optimizasyonu Optimization of PID Controller Parameters in Wind / PV Energy Systems Using PSO and GA Elektrik - Elektronik Mühendisliği Bölümü Elektrik - Elektronik. 27-29.

Ibrahim, H. E. A., Hassan, F. N., \& Shomer, A. O. (2014). Optimal PID control of a brushless DC motor using PSO and BF techniques. Ain Shams Engineering Journal, 5(2), 391-398. https://doi.org/10.1016/j.asej.2013.09.013

Kim, C. H., Yang, J. H., Lim, D. G., \& Suh, B. S. (2010). An enhanced PID controller for speed control of brushless DC motors based on convex set optimization. IFAC Proceedings Volumes (IFAC-PapersOnline), 8(PART 1), 75-80. https://doi.org/10.3182/20100929-3-ro-4017.00014

Kumar, B., Swain, S. K., \& Neogi, N. (2017). Controller design for closed loop speed control of BLDC motor. International Journal on Electrical Engineering and Informatics, 9(1), 146-160. https://doi.org/10.15676/ijeei.2017.9.1.10

Long, W., Wu, T., Jiao, J., Tang, M., \& Xu, M. (2020). Engineering Applications of Artificial Intelligence Refraction-learning-based whale optimization algorithm for high-dimensional problems and parameter estimation of PV model ș. Engineering Applications of Artificial Intelligence, 89(November 2019), 103457. https://doi.org/10.1016/j.engappai.2019.103457

Lu, H., Zhang, L., \& Qu, W. (2008). A new torque control method for torque ripple minimization of BLDC motors with un-ideal back EMF. IEEE Transactions on Power Electronics, 23(2), 950-958. https://doi.org/10.1109/TPEL.2007.915667

Mirjalili, S., \& Lewis, A. (2016). The Whale Optimization Algorithm. Advances in Engineering Software, 95, 51-67. https://doi.org/10.1016/j.advengsoft.2016.01.008

MOHAN, N., UNDELAND, T. M., \& ROBBINS, W. (2007). Gü̧ Elektroniği Çeviriciler, Uygulamalar ve Tasarım (2. Baskı). Literatür Yayıncılık, Dağıtım, Pazarlama San. ve Tic. Ltd. Şti.

Nasiri, J., \& Khiyabani, F. M. (2018). A whale optimization algorithm (WOA) approach for clustering. Cogent Mathematics \& Statistics, 5(1), 1-13. https://doi.org/10.1080/25742558.2018.1483565

Shekhar, S., Saha, P. K., \& Thakura, P. R. (2020). Optimal PID Tuning of BLDC Drive using LQR Technique. 2, 57-574. https://doi.org/10.1109/icisgt44072.2019.00028

Siostrzonek, T., \& Pirog, S. (2007). Motor the Practical Results1. 1541-1545.

Syed Hussien, S. Y., Jaafar, H. I., Ghazali, R., \& Abdul Razif, N. R. (2015). The effects of auto-tuned method in PID and PD control scheme for gantry crane system. International Journal of Soft Computing and Engineering (IJSCE), (6), 121-125. Retrieved from http://eprints.utem.edu.my/14047/1/\%5B1\%5D_F2492014615.pdf

Tabak, A., Kayabasi, E., Guneser, M. T., \& Ozkaymak, M. (2019). Grey wolf optimization for optimum sizing and controlling of a PV/WT/BM hybrid energy system considering TNPC, LPSP, and LCOE concepts. Energy Sources, Part A: Recovery, Utilization and Environmental Effects. https://doi.org/10.1080/15567036.2019.1668880

Tabak, A., Özkaymak, M., Tahir, M., \& Oktay, H. (2017). Optimization and Evaluation of Hybrid PV/WT/BM System in Different e-ISSN: 2148-2683 
Initial Costs and LPSP Conditions. International Journal of Advanced Computer Science and Applications, 8(11), $123-131$. https://doi.org/10.14569/ijacsa.2017.081116

Tarczewski, T., \& Grzesiak, L. M. (2018). An Application of Novel Nature-Inspired Optimization Algorithms to Auto-Tuning State Feedback Speed Controller for PMSM. IEEE Transactions on Industry Applications, 54(3), 2913-2925. https://doi.org/10.1109/TIA.2018.2805300

Uysal, O. (2010). Ekzotermik (Isı Yayan) Kimyasal Reaktörü Simüle Eden Bir Isıl Sistemin Dinamik Davranışının İncelenmesi. İstanbul Teknik Üniversitesi.

Xu, F., Li, T., \& Tang, P. (2008). A Low Cost Drive Strategy for BLDC motor with low torque ripples. 2008 3rd IEEE Conference on Industrial Electronics and Applications, ICIEA 2008, 1, 2499-2502. https://doi.org/10.1109/ICIEA.2008.4582968

Yu, G. R., \& Hwang, R. C. (2004). Optimal PID speed control of brush less DC motors using LQR approach. Conference Proceedings - IEEE International Conference on Systems, Man and Cybernetics, 1, 473-478. https://doi.org/10.1109/ICSMC.2004.1398343

Zhang, H., Tang, L., Yang, C., \& Lan, S. (2019). Advanced Engineering Informatics Locating electric vehicle charging stations with service capacity using the improved whale optimization algorithm. Advanced Engineering Informatics, 41(May 2018 ), 100901. https://doi.org/10.1016/j.aei.2019.02.006

Zhang, Y., Zhang, L., \& Dong, Z. (2019). An MEA-Tuning Method for Design of the PID Controller. Mathematical Problems in Engineering, 2019. https://doi.org/10.1155/2019/1378783 